\title{
Using Neutral Reactor for Suppression of Secondary Arc Current During Single-Phase Auto-Reclosing Under Different Fault Locations
}

\author{
Mahyar Bolhasani ${ }^{1 *}$, Somayeh Sadat Hashemi Kamangar ${ }^{2}$ \\ ${ }^{1}$ Moshanir, Electrical Engineering Consultant Company, Tehran, Iran. \\ 2 Department of Electrical Engineering, Amirkabir University of Technology, Tehran, Iran. \\ * Corresponding author. Tel.: 0989126997941; email: m.bolhasani@moshanir.co \\ Manuscript submitted July 6, 2014; accepted September 18, 2014. \\ doi: $10.17706 /$ ijcee.2014.v6.860
}

\begin{abstract}
When single-phase grounding fault occurs in high voltage transmission line, secondary arc current and recovery voltage must be suppressed in order to ensure that single-phase auto-reclosing operates reliably and successfully. Automatic single-phase reclosing is used to clear single-phase-to-ground faults, which are about $80 \%$ of the transient faults. In order to have successful fast reclosing, different methods are used to extinguish the arc. One of the common methods is to use a single-phase reactor in the neutral of shunt reactor, when transmission line is compensated with shunt reactors. In this paper, exact system and an exact arc modeling is used for transient simulation of neutral reactor and guidelines for selecting the appropriate amount for the neutral reactor are proposed. then uses EMTP-RV software to simulate suppression effect about different fault point locations toward an example of $400 \mathrm{kv}$ double-ended sources high-voltage transmission line.
\end{abstract}

Key words: Fault point location, neutral reactor, secondary arc current, single-phase auto-reclosing.

\section{Introduction}

In extra- and ultra-high voltage (EHV, UHV) transmission lines, three phase reclosing system could only be used when it is very fast. Reclosing time is set based on arc deionization period in air gap between phase conductor and tower body [1]. For high-voltage transmission systems, short circuit capacity of the system usually increases as system voltage increases. The higher short circuit power results in higher short circuit current and higher ionization of the related arc. It results in higher arc extinction time so that the air gap at the fault point would be deionized before reclosing could be performed [2]. This necessitates that reclosing would be performed after some time delay. However, this delay contradicts with the lower stability margin of higher voltage systems. Therefore, application of three phases reclosing might become a non-practical solution for EHV and UHV systems. Application of automatic single-phase reclosing makes it possible to increase system stability limit as system voltage and short circuit level increase. About $80 \%$ of transmission-line faults are single-phase to ground faults. If arc is extinguished completely before first shot of reclosing, reclosing would be performed successfully. In order to have successful fast reclosing; different methods have been applied to extinguish the arc faster. One of the common methods is to use a single-phase reactor in the neutral of the shunt reactors, when the transmission line is compensated with shunt reactors. By appropriate selection of this reactor, arc current is limited and extinguished faster [3], [4]. This method 
is appropriate mainly for transposed lines, whereas use of shunt reactors and neutral reactor with breakers having switching capability among shunt reactor phases and neutral reactor has been suggested for un-transposed lines at very high voltage levels [1], [5].

Appropriate value for the neutral reactor is usually determined only based on the steady-state analysis without considering exact system and arc models. In this research work, power system and arc are exactly modeled and application of neutral reactor is studied in transient mode. In this paper, system modeling is introduced. Traditional method for determining neutral reactor parameters and arc extinction time based on steady-state analysis are presented. Then, an exact arc modeling using electromagnetic based software is briefly explained, using exact network and arc modeling, neutral reactor performance is investigated for transposed and un-transposed lines. Also based on the results obtained through transient simulations, guidelines for selection of neutral reactor are proposed for transposed and un-transposed lines. In this paper, uses suppression method of shunt reactor with neutral small reactor and simulates suppression effect while fault point occurs in different locations.

\section{System Modeling}

In this research, work on a transmission line between the Iran national grid and Armenia ( $400 \mathrm{kV}$ Shinuhayer-one circuit to Heris and another one to Jolfa) has been selected which has a three-phase 50-MVAR reactor at each end (see Fig. 1). The equivalent networks at two sides of the transmission line are modeled appropriately. Series impedance as well as wave impedance are used to model the networks more precisely.

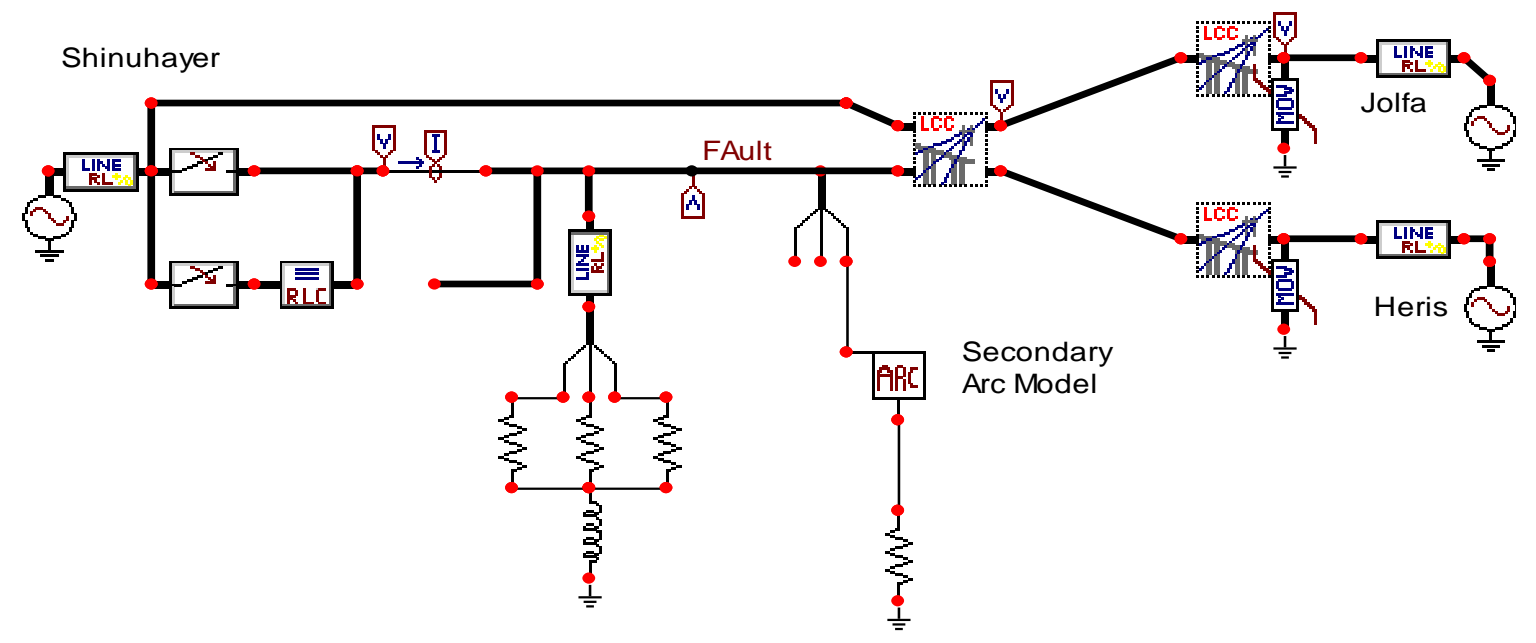

Fig. 1. Shows a single line diagram of the network under study.

\subsection{Transmission Line}

Generally in transient studies, frequency-dependent models are used for exact modeling of transmission lines. The JMarti model is used for modeling the $264-\mathrm{km}$ transmission line under study [3]. This line is transposed symmetrically.

\subsection{Sources}

Equivalent sources impedances have been obtained for Heris, Jolfa and Shinuhayer substations using short circuit studies. Table 1 show positive and zero sequence impedances for equivalent sources in these two substations. A three-phase $200 \Omega$ resistance has been paralleled with each equivalent impedance to model the surge impedance of the sources. This resistance makes the simulations more realistic. 
Table 1. Equivalent Source Impedances

\begin{tabular}{llll}
\hline & Shinuhayer & Jolfa & Heris \\
\hline R1 & 5.44 & 5.44 & 2.72 \\
X1 & 86.56 & 52.48 & 31.68 \\
R0 & 5.28 & 27.68 & 14.4 \\
X0 & 82.4 & 103.84 & 61.28 \\
\hline \hline
\end{tabular}

\subsection{Reactors}

Each 50-MVAR reactor has been modeled by a resistance of $R=10 \Omega$ in series with a reactance of $X=3200 \Omega$ Neutral reactor is modeled by a single-phase reactance. The resistance of neutral reactor is ignored due to its negligible effect.

\section{Traditional Neutral Reactor Selection}

In the traditional method, at first steady-state model of the network is obtained at the arc point, i.e., at the transmission-line end with higher short circuit ratio. This is the worst case which results in maximum secondary arc current. Then, an appropriate amount for reactance of the neutral reactor is selected so that the secondary arc current and voltage are minimized.

\subsection{Secondary Arc Current}

When a single-phase to ground fault occurs, the faulted phase is isolated from both ends by the related protective relays and single pole breakers. In this condition, some voltage is induced in the isolated phase due to capacitive and inductive couplings among the healthy and faulted phases. In spite of the breaker opening at both ends of the faulted phase, the induced voltage feeds current through arc in the faulted point causing arc consistency. This current, called secondary arc current, may be high enough to stop arc extinction after breaker opening. The voltage induced in the faulted phase due to capacitive and inductive coupling is known as secondary arc voltage [1].

\subsection{Appropriate Reactance for Neutral Reactor}

When no shunt and neutral reactors are used, in order to obtain secondary arc current at the worst case (arc resistance equals to zero $\Omega$ ), a single phase to ground short circuit is occurred and capacitive current passing through it can be calculated. Since, major part of the secondary arc current is capacitive and the inductive part is negligible, only the capacitive part is considered in (1)[2].

$$
I_{S}=\frac{E_{a} \cdot w \cdot\left(C_{1}-C_{0}\right)}{3}
$$

where $C_{0}$ : phase to ground capacitor, $C_{1}$ : phase to phase capacitor.

Consistency of the secondary arc current causes continuity of the arc and stops arc extinction. Installation of appropriate reactors (shunt reactor plus neutral reactor) when single-phase auto re-closure is used, could help arc extinction and fast deionization resulting in successful single-phase reclosing.

\subsection{Secondary Arc Extinction Time}

Based on the experience, some equations have been proposed in the past to estimate the arc extinction time. In the estimation of secondary arc extinction time only the stable extinction is taken into consideration, because the instantaneous extinction could happen in less than $0.15 \mathrm{~s}$. According to the experiments, the arc extinction time versus secondary arc for currents about $20 \mathrm{~A}$ and above, and voltage about 20 to $30 \mathrm{kV}$ is calculated from the equation $t_{b}=0.02 . I_{s}$. Furthermore, the dead time of re-closure $t_{d}$ must be $0.25 \mathrm{~s}$ more than $t_{b}$ which results in 


$$
t_{d}=0.25 \cdot\left(0.1 I_{s}+1\right)
$$

The exact value of arc extinction time depends on various factors and parameters of the system and could be better estimated through performing transient studies.

\section{Arc Modeling for Computer Simulation}

It is very difficult to reproduce the real arc behavior by computer simulation due to the extremely random characteristic of the arc. For the accurate arc modeling, the simulation has to consider the functions describing the impulse arc characteristic as well as the recovery of the insulation following the partial extinction using electromagnetic transients analysis. Any arc model requires several input parameters which could be obtained by measurements only. Parameters extracted from measurements can be used to elaborate an arc model which reproduces quite well the main characteristic of that specific arc measurement. Such a model might give less reliable results for other arcing conditions on the same line, or completely inaccurate results for experiments carried out on other lines with different length, voltage level, and conductor arrangement [3]. However, a computer simulation of the process leading to the secondary arc extinction can be a suitable tool for sensitivity studies to identify the main influencing factors and to find the similitude invariants, which are necessary to compile generalized diagrams for the estimation of the arc extinction time [1], [5]. As the purpose of this paper is an investigation of neutral reactor effect and comparison of different amount of its reactance in the cases of transposed and un-transposed lines, [1]. In addition, according to Table 2 , three parameters $u 0, \tau 0$, and $l 0$ are varied around their typical values to generate three different arcs. Existing secondary arc models are based on the differential equation of the arc conductance, which describes the energy balance of the arc column as presented in (5) [1]-[3].

$$
\frac{d g}{d t}=\frac{1}{\tau} \times(G-g)
$$

where $\tau$ is time constant of the arc; $g$ means instantaneous arc conductance; $G$ indicates stationary arc conductance.

The stationary arc conductance can be defined as

$$
G=\frac{\left|i_{\operatorname{arc}}\right|}{\left(u_{0}+r_{0}\left|i_{\operatorname{arc}}\right|\right) \cdot l_{\operatorname{arc}}(t)}
$$

where $l_{\text {arc }}(t)$ is the instantaneous arc length; $u_{0}$ means arc characteristic voltage; $r_{0}$ is the arc characteristic resistance per arc length.

Parameters $\tau, u_{0}$, and $r_{0}$ can be obtained from measurements. The time constant of the arc is inversely proportional to the arc length and can be defined by the following expression:

$$
\tau(l)=\tau_{0}\left(\frac{l_{\text {arc }}}{l_{0}}\right)^{\alpha}
$$

where $\tau_{0}$ : initial time constant; $l_{0}$ : initial arc length; $\alpha$ indicates coefficient in the range of -0.1 to -0.6 .

The time-dependent arc elongation $l_{\text {arc }}(t)$ is the most significant factor that influences the arc extinction and arcing duration. Since, the length of the secondary arc is highly dependent on environmental impacts like wind and thermal buoyancy; it is difficult to consider these random effects accurately in the numerical model. Nevertheless, the above arc model can be used successfully to estimate the maximum arcing duration as worst case or understand the interaction of the secondary arc with the electrical circuit. 


\section{Simulation Studies}

In order to investigate effect of the shunt and neutral reactors on arc extinction time, several simulations have been performed. In all simulations, it is supposed that a single-phase fault (Phase A to G) occurs at 0.0 $\mathrm{s}$ and at $0.1 \mathrm{~s}$ the faulty phase is isolated from both ends by opening the breakers. The arc extinction criterion is considered to be the time when the absolute amount of the arc current amplitude reduces to less than $1 \mathrm{~A}$. As a result, the arc existence period is the arc extinction instant minus $0.1 \mathrm{~s}$. For the modeled real power system, as the short circuit level in Shinuhayer substation is more than Heris and Jolfa substation, arc extinction period in Shinuhayer substation is longer than that of the Heris and Jolfa substations.

Hence, in all simulations to find the worst case, arc is simulated at the Shinuhayer substation, simulation has been performed for both transposed line which the results are shown in Fig. 2, Fig. 4 and un-transposed line which the results are shown in Fig. 3, Fig. 5. According to arc currents is non-sinusoidal and contains harmonics. This is due to the arc nonlinear resistance. When this voltage increases and reaches to the peak of the sinusoid, secondary arc current rises suddenly. Then, by decrease in mutual voltage, due to the arc nonlinear resistance, arc resistance increases suddenly and arc current reduces considerably. This physical process results in a non-sinusoidal current containing considerable amount of harmonics. In this work we change the locations of fault point, and set the distance between initiating terminal and the fault point as $1 \mathrm{~km}, 100 \mathrm{~km}$ to understand the effects of this parameter on results.

In order to investigate effect of the shunt and neutral reactors and fault location on arc extinction time, several simulations have been performed. In all simulations, it is supposed that a single-phase fault (Phase A to G) occurs at $0.0 \mathrm{~s}$ and at $0.1 \mathrm{~s}$ the faulty phase is isolated from both ends by opening the breakers. In this work the effect of neutral reactor on suppression of secondary arc on Heris substation is investigated. In Fig. 2, Fig. 3, Fig. 4, Fig. 5 the arc current are illustrated respectively on un-transposed line with fault location in $1 \mathrm{~km}$ of line(state 1 ), on un-transposed line with fault location in $1 \mathrm{~km}$ of line(state2), transpose line with fault location in $100 \mathrm{~km}$ of line (state3) and un-transpose line with fault location in $100 \mathrm{~km}$ of line(state4). In all simulation we consider the $220 \mathrm{ohm}$ neutral reactor. As it is shown above, if the fault location is changed then it affected on the arc current and changed its amplitude. The comparison of arc extinction time for all states which presented before, is shown in Table 2 .

Table 2. The Comparison of Arc Extinction Time for All States

\begin{tabular}{ccc}
\hline \hline & Arc extinction time for transposed & Arc extinction time for un- transposed \\
& line(sec.) & line(sec.) \\
\hline Fault location $1 \mathrm{~km}$ & 0.55 & 0.7 \\
Fault location $100 \mathrm{~km}$ & 0.3 & 0.42 \\
\hline \hline
\end{tabular}

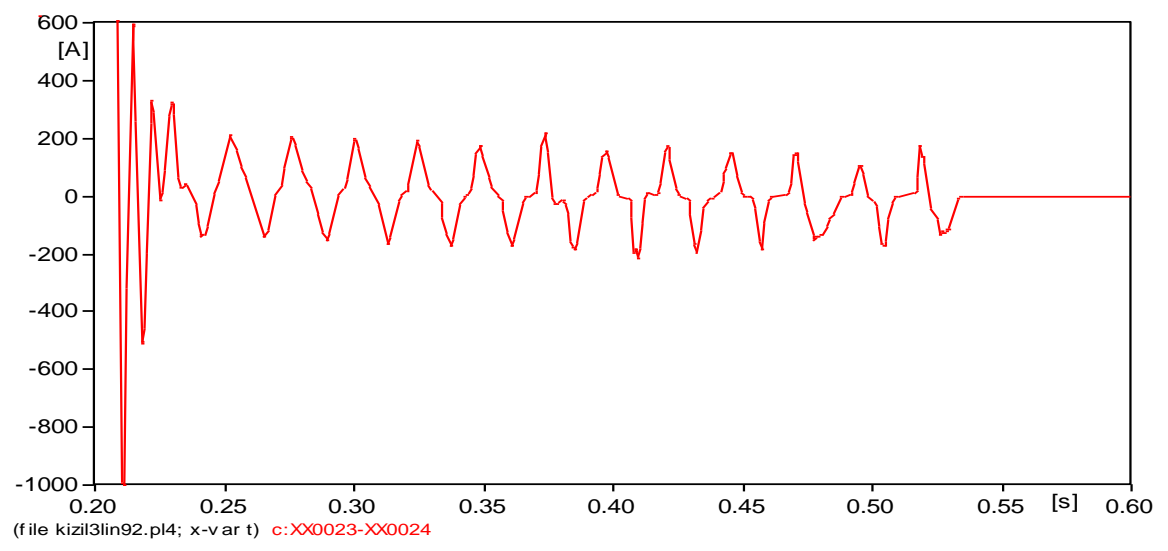

Fig. 2. Secondary arc current transposed line, with shunt compensation with fault location in $1 \mathrm{~km}$ of line. 


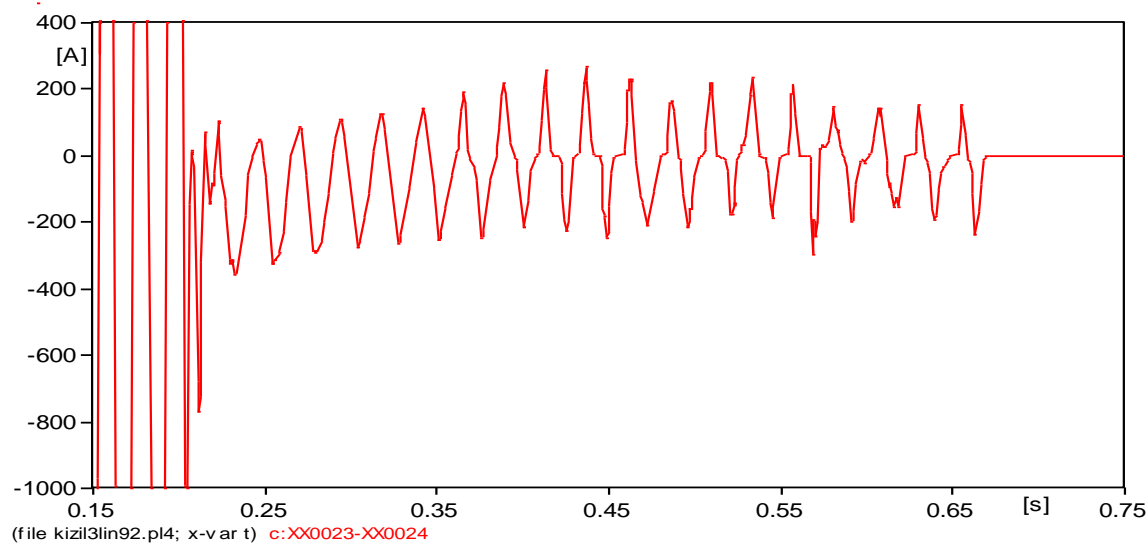

Fig. 3. Secondary arc current un-transposed line, with shunt compensation with fault location in $1 \mathrm{~km}$ of line.

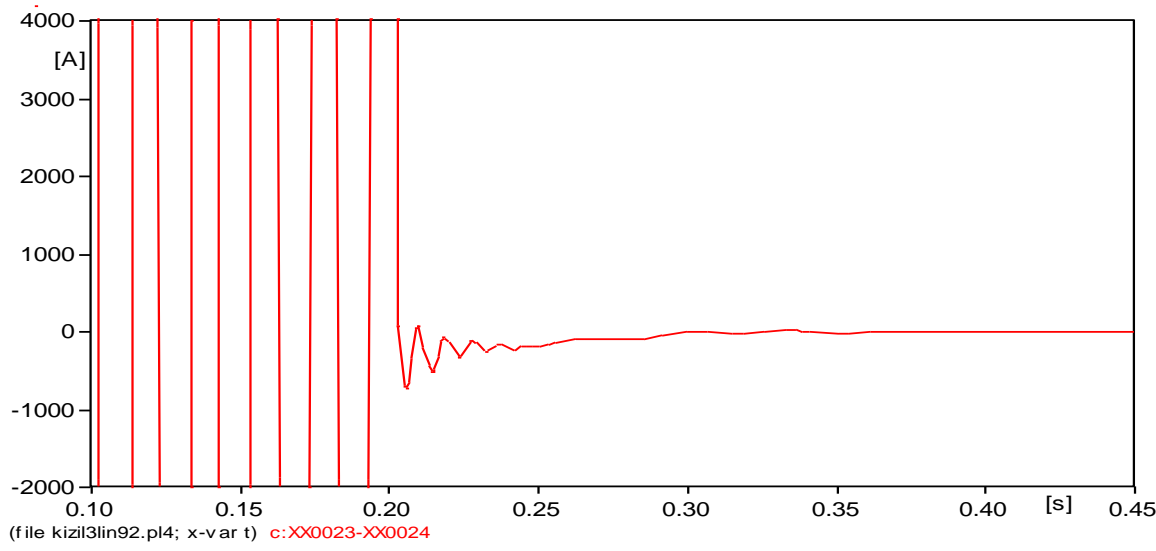

Fig. 4. Secondary arc current transposed line, with shunt compensation with fault location in $100 \mathrm{~km}$ of line.

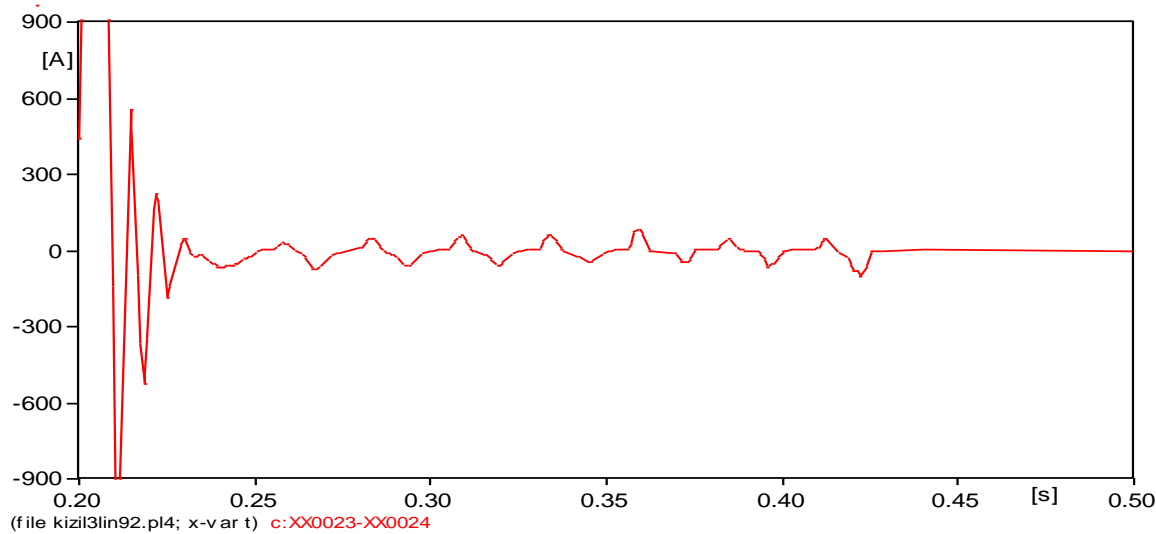

Fig. 5. Secondary arc current un-transposed line, with shunt compensation with fault location in $100 \mathrm{~km}$ of line.

\section{References}

[1] Zadeh, M. R. D., Sanaye-Pasand, M., \& Kadivar, A. (2008, Oct.). Investigation of neutral reactor performance in reducing secondary arc current. IEEE Trans. Power Del., 23(4), 182-192.

[2] Dudurych, I. M., Gallagher, T. J., \& Rosolowski, E. (2004, Apr.). Arc effect on single-phase reclosing time of a UHV power transmission line. IEEE Trans. Power Del., 19(2), 854-860.

[3] Rao, A. S., \& Cook, C. S. (1981, Feb.). Discussion of faults and shunt reactor parameters on parallel resonance. IEEE Trans. Power App. Syst. PAS-100(2), 572-584. 
[4] Shperling, B. R., \& Fakheri, A. (1979, Mar.). Single-phase switching phase parameters for untransposed EHV transmission line. IEEE Trans. Power App. Syst., PAS-98(2), 643-654.

[5] Tran-Quoc, T., Huy, H. V., Duong-La, H., Homg, L. N., \& TrmDinh, L. (1998). Single-phase auto-reclosing of the Vietnam $500 \mathrm{kv}$ system. Proceedings of the International Conference Power System Technology: vol. 2. (pp. 1077 - 1081).

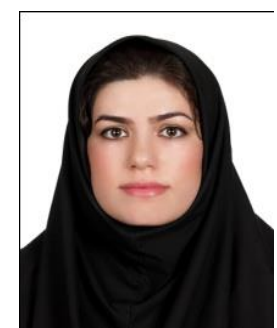

M. Bolhassani received the B.Sc. and M.Sc. degrees in electrical engineering from Tehran Polytechnic and Science \& Research Branch, Islamic Azad University, Tehran, Iran, in 2004 and 2012, respectively.

Her areas of interest include power system protection, control, and analysis and also electric market. She has been working in Moshanir Consultant Company as an electrical engineer, who is mainly working about system study, since 2004 .

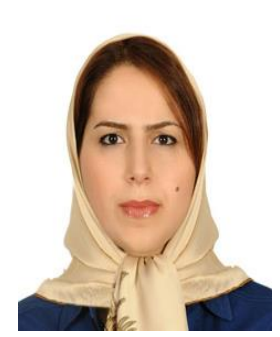

S. Hashemi Kamangar received the B.Sc. and M.Sc. degrees in electrical engineering from Tehran Polytechnic, Tehran, Iran, in 2004 and 2008, respectively.

Her areas of interest include power system protection, control, and analysis and also high voltage. She has been working in Moshanir Consultant Company as an electrical engineer, who is mainly working about system study, since 2004. 